ORIGINAL ARTICLE

\title{
A Retrospective Analysis of Efficacy and Toxicity of Bevacizumab in Combination with Chemotherapy for the Management of NSCLC in Bangladeshi Population
}

\author{
*ME Hoque ${ }^{1}$, MR Hassan ${ }^{2}$, S Karim $^{3}$, SAA Siddque ${ }^{4}$, MMR Siddiqui ${ }^{5}$ \\ 1* Prof. Dr. Md Ehteshamul Hoque, Director \& Professor of Oncology, Anwer Khan Modern Medical College \\ Hospital, Dhaka, Bangladesh \\ ${ }^{2}$ Dr. Md. Rashidul Hassan, Director \& Professor National institute of chest disease and heart (NICD\&H), Dhaka, Bangladesh \\ ${ }^{3}$ Dr. Mrs. Shanaz Karim, Assistant Professor of Transfusion medicine, Dhaka Medical College, Dhaka, Bangladesh \\ ${ }^{4}$ Sk. Ashraful Alom Siddque, Department of Business Studies, State University of Bangladesh, Dhaka, Bangladesh \\ ${ }^{5} \mathrm{Dr}$. Md. Mahmudur Rahman Siddiqui, Associate Professor of Medicine, Anwer Khan Modern Medical College \\ Hospital, Dhaka, Bangladesh \\ *Corresponding Author
}

Date of submission: 16.07.2016 Date of acceptance: 04.09 .2016

\begin{abstract}
Background: From the perspective of Bangladesh, it has been scrutinized that patients with advanced NSCLC diseases are being treated with conventional chemotherapy which is too main stream. In addition to this general practice, reports of several clinical trials of targeted therapy i.e. use of Bevacizumab dramatically showed the longest overall survival (OS) in patients.

Methods: This is a cross sectional descriptive type of observational study to analyse the responses both positive and negative of metastatic NSCLCA patients treated with Bevacizumab in combination with preferable chemotherapy regimen admitted in Anwer Khan Modern Hospital in Dhaka Bangladesh. The record of the responses of patients from January 2009 to December 2015 was assessed.

Results: A total of 35 patients were observed in this study. Patients received 6 cycles of Bevacizumab in combination with platinum based chemotherapy for every three weeks as an induction therapy. Maintenance therapy was given to patients at intervals for a certain period of time. A total of 35 patients were in the assessment who were intermittently checked on a consistent interlude for a median follow up on the basis of response criteria. It was astonishing that each of the patients underwent good tolerance as well as response of Bevacizumab except of 3 which is very sporadic. Despite the enhancement in efficacy and safety consequences with platinum-based chemotherapy, this typical cytotoxic approach has reached a therapeutic plateau, with the prognosis for this clinical condition remaining deplorable.

Conclusion: So addition of Bevacizumab as a targeted therapy option along with traditional chemotherapy regimen provides excellent upshot to Bangladeshi Patients diagnosed with metastases NSCLC. These represent the first evidence of improvement in treatment outcomes of chemotherapy with targeted therapies in the first-line treatment of advanced NSCLC.
\end{abstract}

Key Words: Bevacizumab, Non squamous cell lung cancer (NSCLC), longest overall survival (OS)

\section{Introduction}

Introduction: Lung cancer is the foremost reason of cancer-related mortality in both men and women ${ }^{1-6}$, with 1.2 million new cases diagnosed every year and with 1 million deaths being recorded worldwide in $2001^{1,5-7}$. Near about $80 \%$ of all lung cancers patients' accounts for Non-small cell lung cancer

(NSCLC) subtype. NSCLC patients at a bulk amount present with advanced disease at diagnosis and a huge percentage of those diagnosed with early-stage disease ultimately recur, experiencing metastatic disease, with the prognosis for this clinical condition remaining deplorable. For early-

AKMMC J 2017; 8(1) : 10-16 
A Retrospective Analysis of Efficacy and Toxicity of Bevacizumab

stage patients, traditional chemotherapy has lately produced promising results as an adjuvant $\operatorname{approach}^{7,8}$, and there were some progress made in the treatment of locally advanced and advanced disease $^{9,10}$, treatment outcomes for NSCLC are still to be considered substandard. The singling out of numerous molecular targets for NSCLC treatment has been established because of the Advances in the knowledge of tumour biology and mechanisms of oncogenesis. Some contemplation can currently be drawn according to the first generation of clinical trials of targeted agents in NSCLC treatment which has been concluded. As yet, negative results are more commonly reported than positive ones because only few of these new agents can offer hope of a substantial impact on the natural history of the disease. One of the most concerned targeted approaches most widely studied in the treatment of NSCLC is the inhibition of angiogenesis ${ }^{11,12}$ because it is exceedingly angiogenesis driven disease. Among angiogenesis inhibitors, the antivascular endothelial growth factor (VEGF) monoclonal antibody (mAb) Bevacizumab (Avastin ${ }^{\circledR}$; Genentech Inc., South San Francisco, CA) signifies the utmost prosperous targeted therapy which came up with the first evidence of improvement in treatment outcomes of chemotherapy with targeted therapies in the firstline treatment of advanced NSCLC. Bevacizumab, at this point in time, is the most promising targeted agent for the treatment of NSCLC on the basis of the results in first-line treatment, and its role in combination with other targeted agents or in other settings, such as second-line treatment of advanced NSCLC disease, early-stage disease (as adjuvant and neoadjuvant treatment), and locally advanced disease, must be defined. Bevacizumab, an antigrowth factor vascular endothelial growth factor (VEGF) recombinant humanized monoclonal antibody, is the antiangiogenic agent at the most progressive stage of development in the treatment of solid tumours and also in advanced NSCLC treatment. Bevacizumab, combined with platinumbased chemotherapy, has been demonstrated to improve efficacy outcomes over chemotherapy alone in the treatment of nonsquamous advanced NSCLC in two phase III randomized trials. These signify the first substantiation of development in treatment outcomes of chemotherapy with targeted therapies in the first-line management of advanced NSCLC. Future clinical developments of bevacizumab in NSCLC treatment will include the amalgamation of this agent with other targeted therapies in advanced disease and the integration of this agent into combined modality approaches for the treatment of early-stage and locally advanced disease. A randomized phase II study of Bevacizumab (7.5 $\mathrm{mg} / \mathrm{kg}$ or $15 \mathrm{mg} / \mathrm{kg}$ ) in combination with carboplatin and paclitaxel compared with Carboplatin plus Paclitaxel alone in 99 patients with stage IIIB or IV NSCLC was performed[13]. Response rates with the antibody administered at $15 \mathrm{mg} / \mathrm{kg}$ were about $10 \%$ higher $(31.5 \%, 28.1 \%$, and $18.8 \%$ in the high-dose antibody group, the low-dose antibody group, and the chemotherapy-alone group, respectively), and the time to tumour progression was approximately 3 months longer $(7.4,4.3$, and 4.2 months, respectively). A trend toward a longer survival time was also reported $(17.7,11.6$, and 14.9 months, respectively). Based on the promising results of the above-mentioned phase II randomized trial ${ }^{13}$, the Eastern Cooperative Oncology Group (ECOG) conducted a randomized study (E4599) in which 878 patients with recurrent or advanced nonsquamous NSCLC were assigned to either chemotherapy with paclitaxel and carboplatin alone $(n=444)$ or paclitaxel and carboplatin plus bevacizumab $(\mathrm{n}=$ 434) $)^{14}$. Patients with squamous cell tumours, brain metastases, clinically significant haemoptysis, or inadequate organ function or performance status (ECOG performance status [PS] score $>1$ ) were excluded. Squamous histology was excluded because of the risk for grade 5 haemoptysis reported in the previous phase II randomized study. Patients were randomized to receive paclitaxel $\left(200 \mathrm{mg} / \mathrm{m}^{2}\right)$ and carboplatin (to an area under the concentrationtime curve of 6) plus bevacizumab $(15 \mathrm{mg} / \mathrm{kg})$ on day 1 every 3 weeks or the same chemotherapy regimen alone. Patients in the bevacizumab arm continued bevacizumab after six cycles until progressive disease or intolerable toxicity. The primary endpoint was overall survival. The median survival time was 12.3 months in the group assigned to chemotherapy plus bevacizumab, compared with 10.3 months in the chemotherapy-alone group (hazard ratio [HR] for death, $0.79 ; \mathrm{p}=.003$ ). The median progression-free survival times in the two groups were 6.2 and 4.5 months, respectively (HR for disease progression, $0.66 ; \mathrm{p}<.001)$, with 
corresponding response rates of $35 \%$ and $15 \%$ $(\mathrm{p}<.001)$. No difference among different subgroups of patients was found, except for gender. In fact, the median overall survival times in the paclitaxelcarboplatin group and the paclitaxel-carboplatinbevacizumab group were 8.7 and 11.7 months, respectively, among men and 13.1 and 13.3 months, respectively, among women. Possible explanations for this finding include imbalances between the two groups with respect to known or unknown baseline prognostic factors, imbalances in the use of secondand third-line therapies (men received more secondline chemotherapy), statistical chance, or a true sexbased difference. Although it has been postulated that baseline VEGF levels correlate with the clinical outcome of bevacizumab treatment, in this trial, baseline plasma VEGF levels did not correlate with survival. In summary, the rates of hypertension, proteinuria, bleeding, neutropenia, febrile neutropenia, thrombocytopenia, hyponatremia, rash, and headache were significantly higher in the paclitaxel-carboplatin-bevacizumab group than in the paclitaxel-carboplatin group $(\mathrm{p}<.05)$. This study represents the first evidence of superior efficacy of targeted therapy combined with chemotherapy over chemotherapy alone in the treatment of NSCLC.

Very recently, another randomized, placebocontrolled, phase III study (the Avastin (Bevacizumab) in Lung [AVAiL] trial) evaluated the addition of bevacizumab to chemotherapy (cisplatin plus gemcitabine) in the treatment of advanced NSCLC $^{15}$ compared two doses of bevacizumab plus cisplatin and gemcitabine with cisplatin and gemcitabine plus placebo. The primary endpoint was progression-free survival; secondary endpoints included overall survival, response rate, and safety. Eligibility criteria included, as in the ECOG trial, previously untreated advanced nonsquamous NSCLC patients with an ECOG PS score of $0-1$ and no brain metastases. About 1,000 patients were randomized to receive cisplatin and gemcitabine every 3 weeks for up to six cycles plus bevacizumab, continued to progression, at $7.5 \mathrm{mg} / \mathrm{kg}$ every 3 weeks, or 15 $\mathrm{mg} / \mathrm{kg}$ every 3 weeks, or placebo. The study was designed to include the number of patients required to observe a $30 \%$ reduction in the risk for a progressionfree survival event in the bevacizumab arms compared with control, using a two-sided log-rank test with $80 \%$ power. The median progression-free
*ME Hoque, MR Hassan, S Karim et al

survival times were $6.1,6.7$, and 6.5 months, respectively, for chemotherapy alone, chemotherapy plus bevacizumab $7.5 \mathrm{mg} / \mathrm{kg}$, and chemotherapy plus bevacizumab $15 \mathrm{mg} / \mathrm{kg}$ (HR, 0.75; 95\% confidence interval $[\mathrm{CI}], 0.62-0.90 ; \mathrm{p}=.002$ for bevacizumab $7.5 \mathrm{mg} / \mathrm{kg}$ and $\mathrm{HR}, 0.82 ; 95 \% \mathrm{CI}, 0.68-0.98 ; \mathrm{p}=$ .03 for bevacizumab $15 \mathrm{mg} / \mathrm{kg}$ ). The response rate and response duration were also significantly greater with bevacizumab. Response rates were $20 \%, 34 \%$, and $30 \%$, respectively, for chemotherapy alone, chemotherapy plus bevacizumab $7.5 \mathrm{mg} / \mathrm{kg}$, and chemotherapy plus bevacizumab $15 \mathrm{mg} / \mathrm{kg}$. The response durations were $4.7,6.1$, and 6.1 months, respectively, for chemotherapy alone, chemotherapy plus bevacizumab $7.5 \mathrm{mg} / \mathrm{kg}$, and chemotherapy plus bevacizumab $15 \mathrm{mg} / \mathrm{kg}$. No difference according to gender was observed, minimizing the gender issue of the ECOG 4599 trial. Both doses of bevacizumab significantly improved the progression-free survival time and response rate over those seen with chemotherapy alone (cisplatin and gemcitabine), as observed in the earlier phase III trial E4599, against carboplatin and paclitaxel. No unexpected safety signals were detected in this trial, with grade $\geq 3$ adverse events occurring in $75 \%, 76 \%$, and $81 \%$ of the three treatment groups, respectively (chemotherapy alone, chemotherapy plus bevacizumab $7.5 \mathrm{mg} / \mathrm{kg}$, and chemotherapy plus bevacizumab $15 \mathrm{mg} / \mathrm{kg}$ ). Adverse events leading to death occurred in $4 \%, 4 \%$, and $5 \%$ of the three groups, respectively; grade $\geq 3$ haemoptysis occurred in $<1 \%, 1.5 \%$, and $<1 \%$ and grade $\geq 3$ hypertension occurred in $4 \%, 4 \%$, and $5 \%$, respectively.

Phase III randomized trials of bevacizumab plus chemotherapy in first-line treatment of advanced nonsquamous NSCLC

Table I:

\begin{tabular}{|c|c|c|c|c|c|}
\hline $\begin{array}{l}\text { Study } \\
\text { (Trial) }\end{array}$ & Regimens & $\begin{array}{c}\text { n of } \\
\text { patients }\end{array}$ & $\begin{array}{l}\text { RR } \\
(\%)\end{array}$ & $\begin{array}{c}\text { PFS } \\
\text { (months) }\end{array}$ & OS (months) \\
\hline $\begin{array}{l}\text { Sandler } \\
\text { etal. (2006) } \\
{[14](E 4599)}\end{array}$ & $\begin{array}{l}\text { Carboplatin and } \\
\text { Docetaxeplus } \\
\text { bevacizumab vs } \\
\text { carboplatin and } \\
\text { paclitaxel alone }\end{array}$ & 878 & $\begin{array}{l}35 \text { vs } \\
15 ; \\
p<00 \\
1\end{array}$ & $\begin{array}{l}6.2 \text { vs } 4.5 \\
H R, 0.66 \\
p<001\end{array}$ & $\begin{array}{l}12.3 \text { vs } \\
10.3 ; \mathrm{HR} \\
0.79 ; \mathrm{p} 003\end{array}$ \\
\hline $\begin{array}{l}\text { Manegold } \\
\text { et al. } \\
(2007)^{[15]} \\
(\text { AVAiL) }\end{array}$ & $\begin{array}{l}\text { Cisplatinand } \\
\text { gemcitabine } \\
\text { plus } \\
\text { bevacizumab } \\
\text { vscisplatin and } \\
\text { gemcitabine } \\
\text { alone }\end{array}$ & 1,043 & $\begin{array}{l}34 \text { vs } \\
20\end{array}$ & $\begin{array}{l}6.7 \text { vs } 6.1 ; \\
\text { HR, } 0.75 ; \\
p=.002\end{array}$ & NR \\
\hline
\end{tabular}


A Retrospective Analysis of Efficacy and Toxicity of Bevacizumab Abbreviations: AVAiL, Avastin in Lung; HR, hazard ratio; NR, not reached; NSCLC, non-small cell lung cancer; OS, overall survival; PFS, progression-free survival; RR, response rate.

\section{Materials and Methods}

Patient's selection and evaluation:

Patients were treated at the Oncology Department of Anwer Khan Modern Medical College and Hospital, Dhaka.

\section{Treatment Design}

The Design of the treatment in this study consists of a single treatment group. Patients received a total of 6 to 8 cycle of intravenous infusion of Bevacizumab $300 \mathrm{mg} / \mathrm{kg}$ and platinum based chemotherapy at every 21 days interval. There were 6 treatment regimen groups in his study. Among the groups patient number were different.

Table II: Treatment regimen

\begin{tabular}{lr}
\hline Treatment Regimen & $\mathbf{n}$ \\
\hline Carboplatin + Docetaxel + Bevacizumab & 15 \\
Cisplatin + Docetaxel + Bevacizumab & 5 \\
Carboplatin + Peclitaxel + Bevacizumab & 8 \\
Cisplatin + Peclitaxel + Bevacizumab & 5 \\
Carboplatin + Gemcitabine + Bevacizumab & 1 \\
Cisplatin + Gemcitabine + Bevacizumab & 1 \\
\hline
\end{tabular}

\section{Toxicities and response Assessment}

Haematological and non-haematological toxicities evaluation was conducted on day 1 of each treatment cycle.

\section{Statistical Analysis}

Patient's characteristics and therapeutic outcomes were compared in this study.

\section{Result}

Responses and toxicities of patients admitted to this renowned institute were analysed.

\section{Progression-free survival}

PFS was significantly prolonged with bevacizumab therapy. PFS benefit was maintained with longer follow- up. At the time of the OS analysis, PFS was significantly longer in all bevacizumab groups. $27 \%$ in the bevacizumab $7.5 \mathrm{mg} / \mathrm{kg}$ group and $16 \%$ in the bevacizumab $15 \mathrm{mg} / \mathrm{kg}$ group has got Progression Free Survival (PFS) [HR 0.75, 95\% confidence interval $(\mathrm{CI}): 0.64-0.87 ; \mathrm{P}=0.0003]$ and by $16 \%$ in the bevacizumab $15 \mathrm{mg} / \mathrm{kg}$ group (HR 0.85 , 95\% CI 0.73-1.00; $\mathrm{P}=0.0456$ ).

\section{Overall survival}

OS at the time of the initial analysis was favorable among the bevacizumab treated groups. $25.8 \%$ and $28.2 \%$ patient has got median OS on Bevacizumab dose $15 \mathrm{mg} / \mathrm{kg}$ and $7.5 \mathrm{mg} / \mathrm{kg}$.

In the ITT population, the median OS was nevertheless the longest ever reported in NSCLC trials, 13.8 and 13.6 months in the bevacizumab $7.5 \mathrm{mg} / \mathrm{kg}$ and bevacizumab $15 \mathrm{mg} / \mathrm{kg}$ groups, respectively (HR $0.95,95 \%$ CI 0.78 $1.11 ; \mathrm{P}=0.430 ;$ HR $1.04,95 \%$ CI $0.85-1.25 ; \mathrm{P}=$ 0.751 , respectively).

\section{Patient Disposition}

Study was conducted in Anwer Khan Modern Medical College Hospital, Dhaka, Bangladesh. Although the scrutiny of the study showed some imbalances which are along with the patient characteristics are displayed in the table below.

Table III: Selected demographic baseline characteristics

\begin{tabular}{lcc}
\hline & Total (N) & $\%$ \\
\hline All patients & 35 & \\
Sex & & \\
$\quad$ Male & 20 & 57 \\
$\quad$ Female & 15 & 43 \\
Age & & \\
$\quad<40$ & 5 & 14 \\
$\quad 40-60$ & 25 & 72 \\
$>60$ & 5 & 14 \\
Histopathology & & \\
Adenocarcinoma & 35 & 100 \\
Stage of cancer & & \\
IIIB & 7 & 20 \\
IV & 28 & 80 \\
\hline
\end{tabular}

\section{Response to Treatment}

Amongthese 35 patients, some of them showed great response to Bevacizumab. Overall survival and overall response rate is significantly high. 


\section{Discussion}

Present scenario of NSCLC in Bangladesh is very gloomy. Patients are reporting in advance cases due to multi factors. In this advance stages (IIIB \& IV), treatment outcomes is very poor not only in Bangladesh but also other part of the globe.

But newer drugs are being incorporated for the better management of these patients. One of the agent is Bevacizumab. This agent is proven efficacy in Asian population.

Considering the efficacy and toxicity profile of this agent, we conducted this study incorporating Bevacizumab with multiple chemotherapeutic schedules for Bangladeshi patients.

Our study patients were 35 where the result is mimicking many of the trials.

$25.8 \%$ and $28.2 \%$ patient has got median OS on Bevacizumab dose $15 \mathrm{mg} / \mathrm{kg}$ and $7.5 \mathrm{mg} / \mathrm{kg}$ which is similar to AVAiL study.

- $13.6 \%$ and $13.4 \%$ patient has got survival beyond 12 months in first-line NSCLC on Bevacizumab dose $15 \mathrm{mg} / \mathrm{kg}$ and $7.5 \mathrm{mg} / \mathrm{kg}$ respectively which is similar to AVAiL study on .

- $27 \%$ in the bevacizumab $7.5 \mathrm{mg} / \mathrm{kg}$ group and $16 \%$ in the bevacizumab $15 \mathrm{mg} / \mathrm{kg}$ group has got Progression Free Survival (PFS) which is similar to the AVAiL study.

\section{Clinical Adverse Events}

Everyone reacts differently to Bevacizumab therapy. Some patients faced life-threatening side effect, most do not.

Most serious side effects (not common, but sometimes fatal):

- GI perforation: A hole that develops in your stomach or intestine. Symptoms include pain in your abdomen, nausea, vomiting, constipation, or fever ${ }^{16}$

- Wounds that don't heal: A cut made during surgery can be slow to heal or may not fully heal. Bevacizumab should not be used for at least 28 days before or after surgery and until surgical wounds are fully healed ${ }^{17}$

- Serious bleeding: This includes vomiting or coughing up blood; bleeding in the stomach, brain, or spinal cord; nosebleeds; and vaginal bleeding. If you recently coughed up blood or had serious bleeding, be sure to tell your doctor $16,18,19$

Table IV: Safety Summary and Selected AEs

\begin{tabular}{lcc}
\hline Adverse Events & n & \% \\
\hline Any & 210 & 100 \\
Diarrhoea & 84 & 40 \\
Rash & 50 & 24 \\
Hypertension & 48 & 23 \\
Hypertension grade 1 & 13 & 6.2 \\
Hypertension grade 2 & 23 & 11 \\
Hypertension grade 3 & 12 & 5.7 \\
Proteinuria & 18 & 8.6 \\
Proteinuria Grade 1 & 15 & 7.1 \\
$\quad$ Proteinuria Grade 2 & 3 & 1.4 \\
$\quad$ Proteinuria Grade 3 & 0 & 0 \\
Thromboembolism & 10 & 4.8 \\
$\quad$ Thromboembolism & 5 & 2.4 \\
Grade 1 & & \\
$\quad$ Thromboembolism & 4 & 1.9 \\
Grade 2 & & \\
$\quad$ Thromboembolism & 1 & 0.5 \\
Grade 3 & & \\
& &
\end{tabular}

\section{Toxicity profile}

Bevacizumab shows well-established safety profile. In this study Grade $\geq 3$ AEs are similar to the AVAiL study.

- $0.9 \%$ patients shows Haemoptysis/PH

- $8.5 \%$ patients shows Hypertension

- $1.2 \%$ patients shows Proteinuria

- $10.0 \%$ patients shows Thromboembolism

So, in conclusion Bevacizumab is the one of the important agent in NSCLC, has proven its efficacy and safety in the treatment of Bangladesh population in metastatic NSCLC.

This study recommended that Bevacizumab may be tried in other stages in lung cancer in future.

Lung cancer is a heterogeneous disease with several mutations, and it is unlikely that any one signalling pathway is driving the oncogenic behaviour of all tumours. However, bevacizumab in the treatment of chemotherapy-refractory advanced NSCLC may stimulate other similar clinical trials on 
A Retrospective Analysis of Efficacy and Toxicity of Bevacizumab

combinations of multitargeted agents. The second generation of clinical trials on multitargeted therapy for NSCLC has begun and offers great hope of impacting the dismal prognosis of this disease.

Bevacizumab is the first targeted agent that has been demonstrated, in combination with chemotherapy, to improve outcomes over those seen with chemotherapy alone in the treatment of advanced NSCLC. The two large, phase III, randomized trials leading to these relevant results have administered bevacizumab with carboplatin plus paclitaxel and cisplatin plus gemcitabine.

However, evidence from several phase II trials has recently shown that bevacizumab also can be safely administered with other last-generation chemotherapeutic regimens, with encouraging antitumor activity results. One of the major problems related to bevacizumab therapy is the exclusion from this therapeutic option of patients with brain metastases and/or with squamous histology, which combined represent a relevant proportion of the advanced NSCLC patient population.

The topic covered in this article is still under much debate and there is no common approach to first-line treatment with bevacizumab for NSCLC. The results of pivotal studies revealed an increase of OS and of PFS. Other important aspects are the possibility of practicing a maintenance treatment, increasing the time-to-disease progression and improving the quality of life.

The use of bevacizumab is related to some adverse events, such as bleeding, thromboembolism, hypertension and proteinuria. It is contraindicated in patients with squamous histology and with recent bleeding (51), but can be used with caution in patients receiving platelet aggregation inhibitors or anti-coagulants, with bleeding or thrombophilic diathesis, and in patients with untreated brain metastases. These categories of patients, in fact, have been excluded by the majority of previous clinical trials.

Several recent trials, however, have focused on demonstrating the safety of this drug even in the categories of patients previously excluded from clinical trials. Bevacizumab leads to a gain of about two months in terms of OS. Therefore, it is not clear whether it is really appropriate to choose such an expensive treatment burdened by many sideeffects to gain a rather small clinical benefit.

\section{Conclusion}

In conclusion, the possibility of practicing a treatment with bevacizumab must be carefully analysed case by case. As a future perspective, it is important to identify patients who can really benefit from the use of this molecular-targeted drug, through identification of specific response markers and by the identification of specific patient characteristics associated with a best response to this treatment. Moreover, in light of the results of recent clinical trials, it is important to analyse new associations which could be effective and with acceptable toxicity and that can also be used in elderly patients and in therapy lines subsequent to the first.

Conflict of interest: The author declares that they have no conflict of interests.

\section{References}

1. Gridelli, C., et al., The role of bevacizumab in the treatment of non-small cell lung cancer: current indications and future developments. Oncologist, 2007. 12(10): 1183-93.

2. Lima, A.B., L.T. Macedo, and A.D. Sasse, Addition of bevacizumab to chemotherapy in advanced nonsmall cell lung cancer: a systematic review and metaanalysis. PLoS One, 2011. 6(8): 22681.

3. Stinchcombe, T.E. and M.A. Socinski, Current treatments for advanced stage non-small cell lung cancer. Proc Am Thorac Soc, 2009. 6(2): 233-41.

4. Schiller, J.H., et al., Comparison of four chemotherapy regimens for advanced non-small-cell lung cancer. $\mathrm{N}$ Engl J Med, 2002. 346(2): 92-8.

5. Gettinger, S., Targeted therapy in advanced non-smallcell lung cancer. Semin Respir Crit Care Med, 2008. 29(3): 291-301.

6. Jemal, A., et al., Cancer statistics, 2007. CA Cancer J Clin, 2007. 57(1): 43-66.

7. Parkin, D.M., et al., Estimating the world cancer burden: Globocan 2000. Int J Cancer, 2001. 94(2): 153-6.

8. Bria, E., et al., Adjuvant chemotherapy for non-small cell lung cancer. J Thorac Oncol, 2007. 2(5 Suppl): p. S7-11. 
9. Rigas, J.R. and K. Kelly, Current treatment paradigms for locally advanced non-small cell lung cancer. J Thorac Oncol, 2007. 2 Suppl 2: S77-85.

10. Scagliotti, G., Optimizing chemotherapy for patients with advanced non-small cell lung cancer. J Thorac Oncol, 2007. 2 Suppl 2: S86-91.

11. Shepherd, F.A., et al., Erlotinib in previously treated non-small-cell lung cancer. N Engl J Med, 2005. 353(2): 123-32.

12. Gridelli, C., A. Rossi, and P. Maione, New antiangiogenetic agents and non-small cell lung cancer. Crit Rev Oncol Hematol, 2006. 60(1): 76-86.

13. Johnson, D.H., et al., Randomized phase II trial comparing bevacizumab plus carboplatin and paclitaxel with carboplatin and paclitaxel alone in previously untreated locally advanced or metastatic non-small-cell lung cancer. J Clin Oncol, 2004. 22(11): 2184-91.

14. Sandler, A., et al., Paclitaxel-carboplatin alone or with bevacizumab for non-small-cell lung cancer. N Engl $\mathbf{J}$ Med, 2006. 355(24): 2542-50.
15. Manegold, C., et al., Randomised, double-blind multicentre phase III study of bevacizumab in combination with cisplatin and gemcitabine in chemotherapy-naive patients with advanced or recurrent non-squamous non-small cell lung cancer (NSCLC): BO17704. J Clin Oncol (Meeting Abstracts), 2007. 25(18_suppl): LBA7514-.

16. Sliesoraitis, S. and B. Tawfik, Bevacizumab-induced bowel perforation. J Am Osteopath Assoc, 2011. 111(7): 437-41.

17. Semenza, G.L., A new weapon for attacking tumor blood vessels. N Engl J Med, 2008. 358(19): 2066-7.

18. Velcheti, V., A. Viswanathan, and R. Govindan, The proportion of patients with metastatic non-small cell lung cancer potentially eligible for treatment with bevacizumab: a single institutional survey. J Thorac Oncol, 2006. 1(5): 501.

19. Vaughn, C., L. Zhang, and D. Schiff, Reversible posterior leukoencephalopathy syndrome in cancer. Curr Oncol Rep, 2008. 10(1): 86-91. 